\title{
Drowsy Driving Prevention Service Using EEG
}

\author{
Jeong Hee Hwang ${ }^{1}$, Soon Won $\mathrm{Ahn}^{2}$, Ji Yeon $\mathrm{Kim}^{3}$ and Jeong Hee Chi ${ }^{4 *}$ \\ ${ }^{1}$ Department of Computer Science, Namseoul University, Korea \\ ${ }^{2,3,4}$ Department of Software, Konkuk University, Korea \\ 1jhhwang@nsu.ac.kr, ${ }^{2,3,4}\{j h c h i$, bri0337, sarakjykjy\}@konkuk.ac.kr
}

\begin{abstract}
This paper proposes a system which can prevent drowsy driving. By analyzing brain waves measured by NeuroSky MindWave headset, the system can serve to prevent drowsy driving. It also provides driver with drowsy driving pattern by accumulating environmental elements like weather, temperature, and location, etc. in which the driver habitually falls drowsy. By analyzing brain wave data, the system creates a classification model based on deep neural network to determine whether the driver is drowsy or not. When it determines that the driver is drowsy, it sends alarm to the driver's smart phone or smart watch, or to the device of passenger designated by the driver beforehand. We expect that the system will help prevent drowsy driving.
\end{abstract}

Keywords: Brain wave, EEG, Drowsiness, Driving Habit

\section{Introduction}

According to the Korea Highway Corporation, drowsiness driving is the most common cause of death by traffic accidents [1,2]. Currently, while various methods by the Korea Highway Corporation are used to prevent drowsy driving such as pinwheel on the roadside, banner, siren, and rumble strips, highway drowsiness shelter [2]. However, they have limits in the sense that they cannot directly wake drowsy drivers up. And, commercialized technologies related with driving assistant system such as the system maintaining distance between cars, and the lane departure warning system $[3,4]$ are the services to deal with the results of drowsy driving, and they cannot prevent drowsy driving. Therefore, it is important to develop a system which can detect drowsy driving immediately and warn the driver of it.

The previous studies related to drowsiness have been carried out from decades ago [57]. Recently, development of systems to detect drowsiness of driver and warn the driver of it has emerged as an important issue in autonomous driving research [8-12]. Recent some studies focus on finding the causes of drowsiness and eradicating them, such as the essence of drowsiness and characteristics of sleep-related disease patients and effects of treatment of those diseases [13-15]. Also, several recent studies have suggested driver drowsy detection or warning system using camera [16-21]. These systems recognize the blinking pattern of the eyes and the movement of the head through face recognition from the camera to recognize the drowsiness. However, camera-based methods have a problem that the measurement method is affected by the brightness of the driver's seat. And, there are researches on methods using bio-signals such as analysis of characteristics of differences in heart beats using ElectroCardioGram(ECG), and brain wave using ElectroEncephaloGraphy(EEG) [22-25]. The existing studies using EEG are difficult to recognize accurate drowsiness by using eye blink information measured when EEG information is measured [25].

Received (January 10, 2018), Review Result (March 26, 2018), Accepted (July 12, 2018)

* Corresponding Author 
This paper suggests a Driver-Waking system (DW system), which can provide driver with a system which can detect drowsiness of driver by measuring EEG, and wake driver up and the pattern where the driver tends to get drowsy using accumulated EEG data and information on the environmental conditions surrounding the driver. If the DW system decides that the driver is drowsy by the measurement of alpha wave, beta wave, theta wave, and delta wave of brain, it wake the driver up by sending alarm to the driver's smart phone or smart watch, or notifying it to the passenger if the driver is drowsy. EEG measured while the driver is drowsy are stored along with other information like weather, temperature, location of the car, day and time when the car is driven, and season, and the data is used to provide the pattern when driver fall drowsy.

The remainder of the paper is organized as follows: Section 2 reviews researches related to the proposed system, and in Section 3 describes the architecture of proposed system in detail, Section 4 shows the simulation results for the proposed system. We conclude the work in Section 5.

\section{Related Works}

The researches detecting drowsiness can be divided into two methods: what uses biosignal, and what uses video treatment. Bio-signals are generated by human body such as EEG, pulse, and temperature, etc. In order to get bio-signals, it is necessary to attach such devices to human body [22-25]. While it is cumbersome, we can relatively accurately measure whether the driver is drowsy or not. In contrast, the method based on video treatment is convenient because it only needs video signals input by camera [16-21].

When driver is tired, they cannot maintain a high awake condition. This paper is focusing on drowsiness driving based on EEG data. The EEG when awake or sleepy is consistently related with changes of alpha wave and theta wave of EEG. EEG analysis is mainly observation of spectrum components. Based on spectrum analysis, many studies have found that delta wave, theta wave, alpha wave, and beta wave are related with drowsy [26-28].

Brain wave is electric signal of electric activity of brain measured using electrode attached on the surface of head [27, 28]. As EEG are shown as a complex pattern of waves, it is impossible to visually observe wave types of it. In general, to observe EEG, power spectrum analysis method is used. This method assumes that EEG is linear combination of simple tremors having specific frequencies, classifies waves into frequency components, and expresses the sizes or powers of them.

In general, depending on the ranges of trembling frequency, EEG are classified into $\delta$ wave $(0.2 \sim 3.99 \mathrm{~Hz}), \theta$ wave $(4 \sim 7.99 \mathrm{~Hz}), \alpha$ wave $(8 \sim 12.99 \mathrm{~Hz}), \beta$ wave $(13 \sim 29.99$ $\mathrm{Hz}$ ), and $\gamma$ wave $(30 \sim 50 \mathrm{~Hz})[29]$. $\delta$ wave appears to adult while one is in deep sleep, or to newly born baby. $\alpha$ wave mainly appears when one is comfortable without tension. In particular, $\alpha$ wave appears when one closes one's eyes and feel stable, but, if one opens eyes and looks at an object, or when one is excited, $\alpha$ wave is suppressed. $\theta$ wave mainly appears when one is stable or goes to sleeping condition. $\beta$ wave mainly appears on prefrontal part of brain and appears when one is consciously active such as the condition of being awake, or of speaking. It is reported that $\gamma$ wave appears when one is irritated, or when one treats high-level cognitive information such as abstraction or judgement.

This paper proposes a drowsy driving preventing system through EEG analysis. In the system, NeuroSky MindWave headset continuously monitors EEG to detect drowsiness, and if it decides that driver is drowsy, it wakes them up with alarm.

\section{Driver-Waking System}

We propose the Driver-Waking system (DW system) as drowsy preventing service using EEG. Figure 1 illustrates the overall process of the proposed system. The framework consists of 4 stages. 


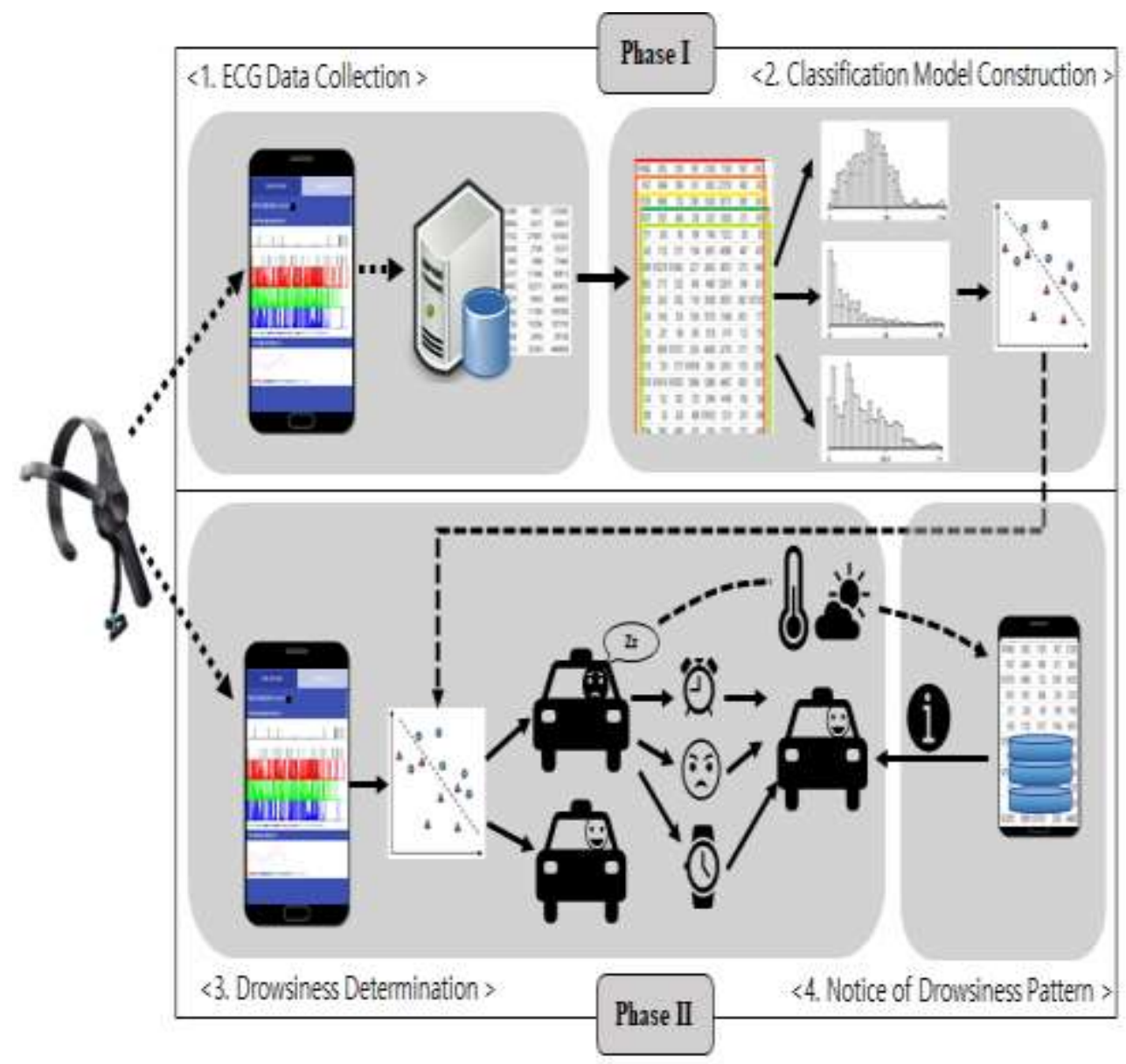

Figure 1. Overall Process of Driver-Waking System

In the first stage, the system collects EEG to analyze the type of EEG when the user is awake and sleepy. The data collected from the headset is stored in the database via the smartphone. In the second step, feature information is extracted from the collected data and a classification model is created. The classification model is generated using Deep Neural Network(DNN) by 24 feature information and labels. In the third stage, it judges whether the driver is drowsy or not through the classification model. Finally, if the driver is judged to be drowsy, generate alarm, information on temperature, weather, and driving location, etc. are saved to give warning to the driver later. The first and second stages are the stages for model generation, and summarized into Phase I. And, the phase where the system judge drowsiness from the generated model, and provides pattern information service is called Phase II.

\subsection{Classification Model Construction}

Phase I in the Driver-Waking system proposed in this paper is the phase of generating the classification model. First, it collects EEG data from participants. The EEG data used for generating a classification model is stored in the following structure.

$$
T_{i, j}=\left\{p_{i}, h-a_{j}, l-a_{j}, h-b_{j}, l-b_{j}, t j, d_{j}, m-g_{j}, l-g_{j}, l_{a b e l_{j}}\right\}
$$

EEG data is stream data which inflows continuously, and can be handled as a transaction in the stream. Transaction $T_{i, j}$ means the data of the $i^{\text {th }}$ participant's $j^{\text {th }}$ EEG data. Information on each transaction are illustrated in Table 1. 
Table 1. Components of $\mathrm{T}_{\mathrm{i}, \mathrm{j}}$

\begin{tabular}{l|l}
\hline Attribute & \multicolumn{1}{|c}{ Description } \\
\hline$p_{i}$ & $i^{\text {th }}$ participant \\
$h-a_{j}$ & $j^{\text {th }}$ high alpha \\
$l-a_{j}$ & $j^{\text {th }}$ low alpha \\
$h-b_{j}$ & $j^{\text {th }}$ high beta \\
$l-b_{j}$ & $j^{\text {th }}$ low beta \\
$t_{j}$ & $j^{\text {th }}$ theta \\
$d_{j}$ & $j^{\text {th }}$ delta \\
$m-g_{j}$ & $j^{\text {th }}$ mid gamma \\
$l-g_{j}$ & $j^{\text {th }}$ low gamma \\
label $j$ & $j^{\text {th }}$ drowsiness or not \\
\hline
\end{tabular}

To collect EEG information on drowsiness per participant, the system measures EEG in awake and drowsy conditions per participant. Such information on drowsiness are added to $l a b e l$, and stored with EEG incoming to the DW system. EEG are continuous stream data, and, applying sliding window, we determined whether one is drowsy or not in every second per $\mathrm{k}$ unit. Figure 2 shows a sliding window with $\mathrm{k}=5$. In this example, we extract feature data for model generation based on EEG measured within each sliding windows.

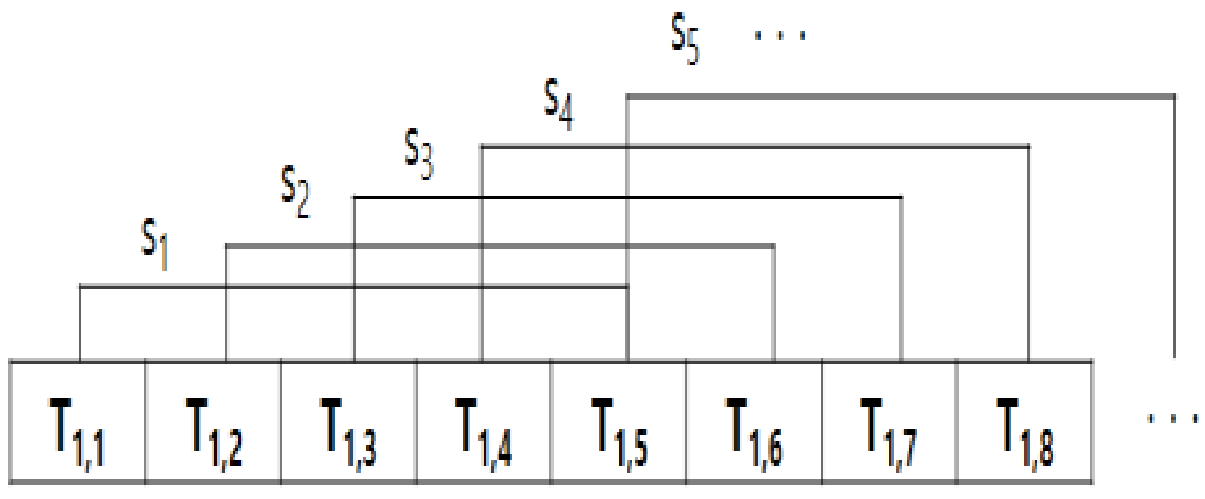

Figure 2. Sliding Window

Figure 3 shows the preprocessing process for extracting the characteristics of the data to generate the data model. Figure 3(a) shows a portion of the dataset. To make classification data model for drowsy, first, the collected data extracts features such as frequency, average, and standard deviation of each EEG for each sliding windows. The frequency is the frequency of high EEG bandwidth in the sliding window as shown in Figure 3(b). Next, the feature data are standardized using mean and standard deviation as shown in Figure 3(c). Three features are extracted for each EEG wave, and all 24 features are extracted as shown in Figure 3(d). 


\begin{tabular}{|c|c|c|c|c|c|c|c|c|}
\hline \multicolumn{1}{c|}{ H-alpha } & L-alpha & H-beta & L-beta & theta & delta & M-gamma & L-gamma \\
\cline { 2 - 10 } & 16745683 & 30502 & 11280 & 9927 & 213585 & 717628 & 15927 & 20412 \\
\hline 14537 & 26664 & 9004 & 6311 & 36653 & 2215192 & 4683 & 2432 \\
\hline
\end{tabular}

(a) Dataset

\begin{tabular}{|c|c|c|c|c|c|c|c|c|}
\hline \multirow{2}{*}{$\begin{array}{c}\text { Sliding } \\
\text { Window }\end{array}$} & \multicolumn{8}{|c|}{ frequency } \\
\cline { 2 - 9 } & H-alpha & L-alpha & H-beta & L-beta & theta & delta & M-gamma & L-gamma \\
\hline $\mathrm{S}_{1}$ & 46 & 39 & 29 & 37 & 66 & 35 & 5 & 13 \\
\hline $\mathrm{S}_{2}$ & 45 & 40 & 29 & 38 & 66 & 35 & 5 & 13 \\
\hline $\mathrm{S}_{3}$ & 45 & 40 & 29 & 38 & 67 & 35 & 5 & 13 \\
\hline $\mathrm{S}_{4}$ & 44 & 41 & 30 & 39 & 67 & 35 & 5 & 13 \\
\hline $\mathrm{S}_{5}$ & 44 & 41 & 30 & 39 & 67 & 35 & 5 & 13 \\
\hline $\mathrm{S}_{6}$ & 45 & 41 & 30 & 39 & 68 & 36 & 5 & 13 \\
\hline$\ldots$ & \multicolumn{7}{|c|}{} \\
\hline
\end{tabular}

(b) Feature Extraction

\begin{tabular}{|c|c|c|c|c|c|c|c|c|}
\hline \multirow{2}{*}{$\begin{array}{l}\text { Sliding } \\
\text { Window }\end{array}$} & \multicolumn{8}{|c|}{ frequency } \\
\hline & H-alpha & L-alpha & H-beta & L-beta & theta & delta & M-gamma & L-gamma \\
\hline $\mathrm{S}_{1}$ & -0.02367 & -2.75714 & -0.75245 & -0.56317 & 0.75653 & 0.38742 & -0.93506 & -1.02216 \\
\hline $\mathrm{S}_{2}$ & -0.2004 & -2.55016 & -0.75245 & -0.34362 & 0.75653 & 0.38742 & -0.93506 & -1.02216 \\
\hline $\mathrm{S}_{3}$ & -0.2004 & -2.55016 & -0.75245 & -0.34362 & 0.83949 & 0.38742 & -0.93506 & -1.02216 \\
\hline $\mathrm{S}_{4}$ & -0.37713 & -2.34319 & -0.64665 & -0.12408 & 0.83949 & 0.38742 & -0.93506 & -1.02216 \\
\hline $\mathrm{S}_{5}$ & -0.37713 & -2.34319 & -0.64665 & -0.12408 & 0.83949 & 0.38742 & -0.93506 & -1.02216 \\
\hline $\mathrm{S}_{6}$ & -0.2004 & -2.34319 & -0.64665 & -0.12408 & 0.92245 & 0.4693 & -0.93506 & -1.02216 \\
\hline$\ldots$ & & & & & & & & \\
\hline
\end{tabular}

(c) Standardization

\begin{tabular}{|c|c|c|c|c|c|c|c|}
\hline \multirow{2}{*}{$\begin{array}{l}\text { Sliding } \\
\text { Window }\end{array}$} & \multicolumn{3}{|c|}{ H-alpha } & \multicolumn{3}{|c|}{ L-alpha } & \multirow{8}{*}{$\ldots$} \\
\hline & frequency & avg & std & frequency & avg & std & \\
\hline $\mathrm{S}_{1}$ & -0.02367 & -0.09175 & 0.06289 & -2.75714 & -2.73458 & -2.96754 & \\
\hline $\mathrm{S}_{2}$ & -0.2004 & -0.25735 & -0.12552 & -2.55016 & -2.53554 & -2.71133 & \\
\hline $\mathrm{S}_{3}$ & -0.2004 & -0.25741 & -0.12549 & -2.55016 & -2.53569 & -2.71126 & \\
\hline $\mathrm{S}_{4}$ & -0.37713 & -0.42382 & -0.31738 & -2.34319 & -2.33719 & -2.45889 & \\
\hline $\mathrm{S}_{5}$ & -0.37713 & -0.42387 & -0.31735 & -2.34319 & -2.33711 & -2.45892 & \\
\hline $\mathrm{S}_{6}$ & -0.2004 & -0.25739 & -0.12572 & -2.34319 & -2.33711 & -2.45892 & \\
\hline$\ldots$ & & & & $\ldots$ & & & \\
\hline
\end{tabular}

(d) Final Datasets

Figure 3. EEG Data Preprocessing 


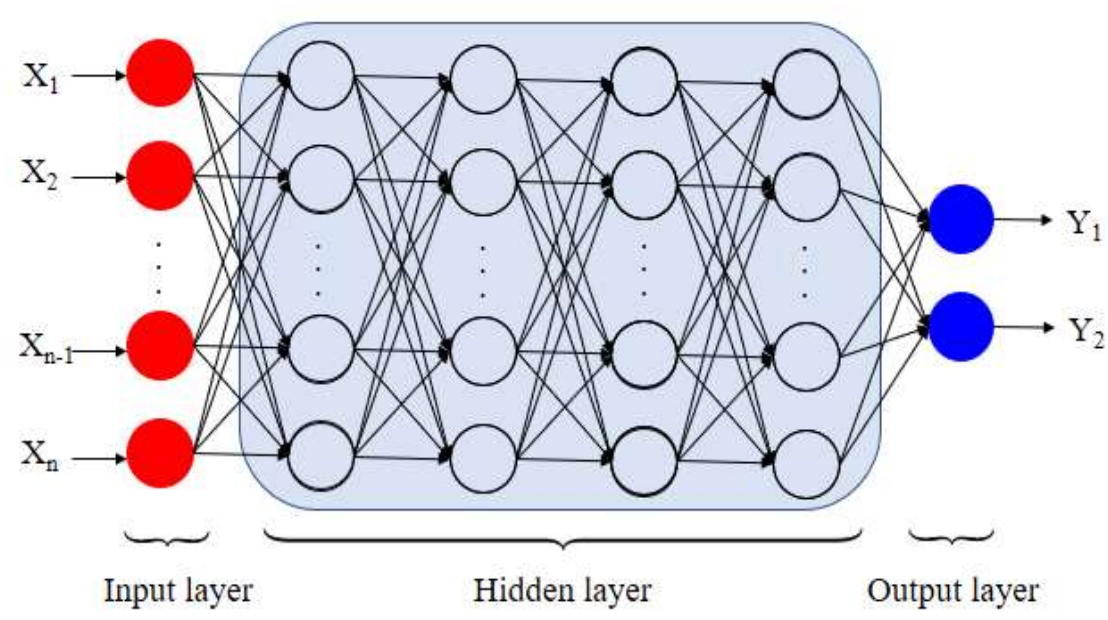

Figure 4. Deep Neural Network Model

After completing data collection and feature extraction, we generated a classification model by feature of EEG data within a sliding window. The classification model was made with Deep Neural Network(DNN) algorithm composed of 4 hidden layers using gradient descent optimizer as shown in Figure 4.

\subsection{Drowsiness Determination}

The second phase of the DW system is to judge drowsiness and prevent drowsiness by generating various events. The system receives EEG information for five minutes, extracts the characteristics of the received data, and uses it as test data for the classification model to determine whether to be drowsy. To provide alarm service to the driver who is determined to be drowsy, the system lets the driver to choose the device to receive the alarm to wake them up. Alarm is sent to smart phone or smart watch of the driver, or it can also be sent to the device of passenger. We also make the system store situational information like the time, weather, and location when the driver is drowsy to manage the pattern where drowsiness tends to occur. Then, the service is constructed to allow the driver to identify their drowsiness patterns when they log in the system.

\section{Simulations}

We constructed the Driving-Waking system which can prevent drowsy driving using EEG. In the experimental environment, the server was based on Java and Python, and the application is Android-based. And we used the NeuroSky MindWave headset. The device is a device that acquires EEG data by wearing like a normal headphone. It has a microchip that pre-processes EEG signals and transmits the data through Bluetooth. The output data is an FFT performed on a signal that provides band power, and these powers are data that are adjusted and filtered relative to each other.

To generate the classification model, we secured 15-minute video clip taken per each of the 8 participants to compare EEG when the driver is drowsy and not. Among the 8 kinds of EEG sent through NeuroSky MindWave headset, we used EEG collected for 5 minutes as standard values to determine drowsiness or not. After 5 minutes, the system performed determination of drowsiness every second.

\subsection{Evaluation of the Classification Model}

We generated a classification model to determine drowsiness of participant using DNN algorithm. 24 features of mean, median and standard deviation were extracted from eight EEG collected from eight participants. Based on such feature, we built the model. To 
evaluate the performance of the classification model, $70 \%$ of the data set was used as training data set, and the remaining 30\% was used as test data set. Learning rate was set up at 0.1 , and we tested by constructing neutral network composed of the fourth layers. Figure 5 shows the test results of the classification model.

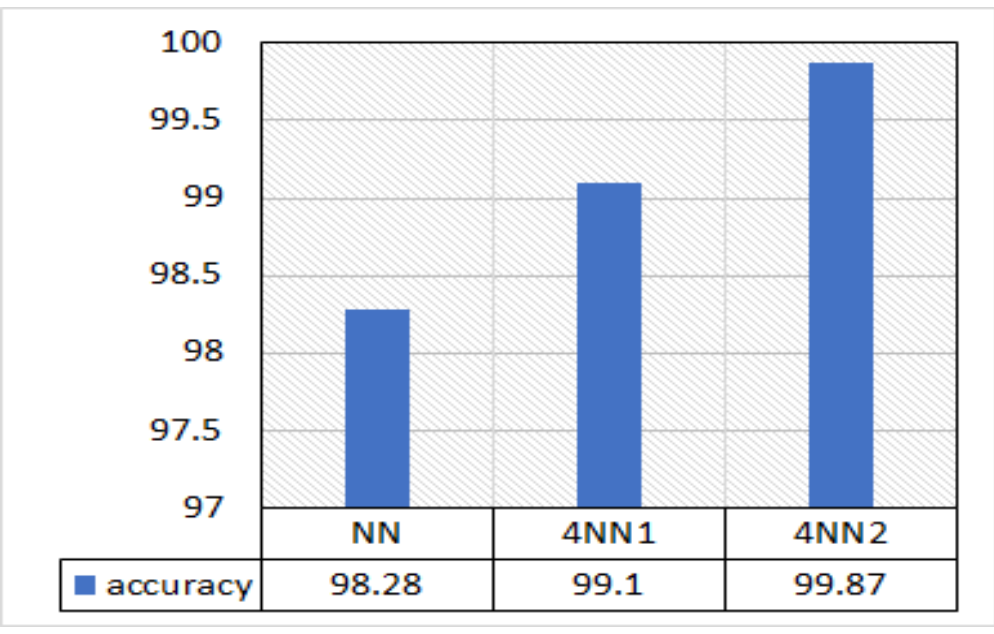

Figure 5. Accuracy of Classification Model

We tested NN and 4NN1 whose epochs were set at 10K, and 4NN2 whose epochs were set at 50k. Tests showed that NN shows accuracy of 98.28, and what was composed of 4 layers showed $99.1 \%$ accuracy. When epochs were increased, accuracy was improved up to $99.87 \%$.

\subsection{MindWake App Service}

The App of DW system, MindWake which collects EEG of participant and provides the driver alarm service was realized as shown in Figure 6. MindWake is based on android. After logging in, the participant could select alarm as shown in Figure 6(a). Then, the system is connected to NeuroSky MindWave headset and Bluetooth. When they are properly connected, the MindWake App can receive EEG data real time as shown in Figure 6(b).

If the system analyzes received EEG, and determines that the driver is drowsy, it generates alarm as shown in Figure 6(c). Alarm rings in smart phone and smart watch in the same type. The system arranges that alarm ends with voice recognition function. To end alarm sound, the participant should say "Hi". If the driver selects passenger as the person who will receive alarm, the alarm message is sent to the passenger, who can wake the drowsy driver up. And, if the driver refers to his or her pattern of drowsiness, the system provides information like the time when driver frequently falls drowsy and weather, etc., to make the driver prevent drowsy driving as shown in Figure 6(d).

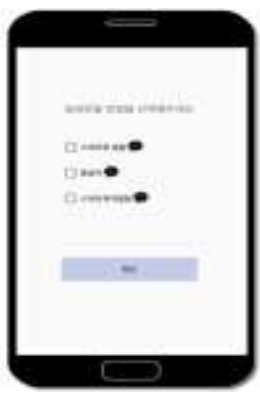

(a) Choice of Alarm

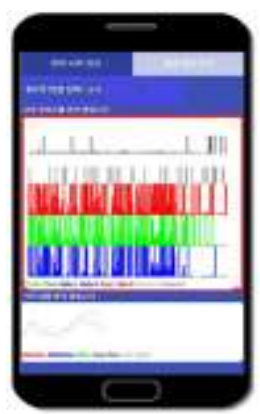

(b) Reception of EEG 


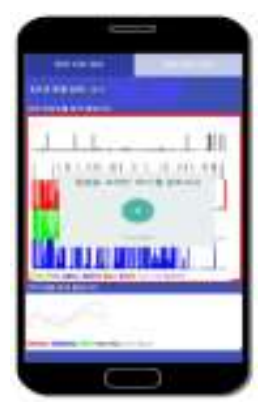

(c) Generating Alarm

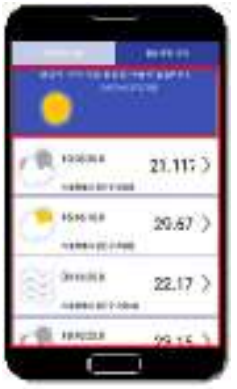

(d) Pattern Reference

Figure 6. Screens Showing how MindWake App is Realized

We tested the system to 10 participants to know whether alarm on drowsiness functions properly. Table 2 shows the test results.

Table 2. Results of App Use

\begin{tabular}{cccc}
\hline participant & wake & drowsy & useful \\
\hline P1 & O & $\bigcirc$ & $\bigcirc$ \\
P2 & X & $\bigcirc$ & $\bigcirc$ \\
P3 & X & $\bigcirc$ & $\bigcirc$ \\
P4 & X & O & O \\
P5 & X & $\bigcirc$ & $\bigcirc$ \\
P6 & X & $\bigcirc$ & $\bigcirc$ \\
P7 & X & X & $\bigcirc$ \\
P8 & X & O & O \\
P9 & X & $\bigcirc$ & $\bigcirc$ \\
P10 & X & $\bigcirc$ & $\bigcirc$ \\
\hline
\end{tabular}

Tests revealed that, while, in the case of P1, alarm ringed when the participant was not drowsy, the alarms did not ring for other participants when they were not drowsy. The alarm responses when participants were drowsy were as follows: P7 said that though he or she was drowsy, the alarm did not ring; P1, P5, and P10 said that when they tried to get drowsy, the alarm ringed. The characteristics of the test makes it difficult to evaluate accuracy of the system. But, all the 10 participants said that the system was useful.

The system proposed in this paper is an app which determines drowsiness of driver using the relatively cheap device measuring EEG and prevents drowsy driving. The system is expected to help prevent safety accidents in industrial sites, drowsiness of learners, and accidents caused by night watches.

\section{Conclusion}

This paper proposed the Driver-Waking system which makes the NeuroSky MindWave headset measure and analyze EEG, and, if it determines that the driver to be drowsy, sends alarm to prevent drowsy driving, and which also provides information on drowsy driving pattern of the driver using situational information like weather, temperature, location, time, season, and date. The system generates classification model by analyzing EEG using DNN algorithm. By monitoring EEG, the system detects when driver gets drowsy, and sends alarm to the driver or a person the driver designated in advance. It is expected that it can help prevent traffic accidents. In addition, it is expected that, by 
providing driver with information on his or her pattern of drowsiness like time, place, and weather, etc., it can also prevent drowsy driving.

\section{Acknowledgments}

This research was supported by the MSIT(Ministry of Science and ICT), Korea, under the Seoul Accord Vitalization Program(IITP-2018-2012-1-00593) supervised by the IITP(Institute for Information \& communications Technology Promotion).

\section{References}

[1] W. Y. Lee and J. S. Oh, "A Study on the Measures to Prevent Sleepy Driving”, Road Traffic Authority, Traffic Science Institute, (2014).

[2] Korea Consumer Agency, "Survey on safety status of highway drowsiness shelter", Safety Report, (2017), pp. 1-38.

[3] T. Sugawara, H. Altmannshofer and S. Kakegawa, "Applications of Road Edge Information for Advanced Driver Assistance Systems and Autonomous Driving", Advanced Microsystems for Automotive Applications, (2018), pp. 71-86.

[4] S. Kakegawa and T. Sugawara, "Road edge detection using dynamic programming with application to free space computation", 25th Aachen Colloquium Automobile and Engine Technology, (2016), pp. 1395-1418.

[5] T. M. Mast, H. V. Jones and N. W. Heimstra, "Effects of fatigue on performance in a driving device", Highway Research Record, vol. 122, (1966).

[6] I. D. Brown, "Car Driving and Fatigue", Triangle (Sandoz Journal of Medical Science), vol. 8, (1967), pp. 131-137.

[7] E. I. Dureman and C. Boden, "Fatigue in simulated car driving", Ergonomics, vol. 15, (1972), pp. 299308.

[8] J. Qiang, Z. Zhiwei and L. Peilin, "Real-Time Nonintrusive Monitoring and Prediction of Driver Fatigue", IEEE Trans. on Vehicular Technology, vol. 53, no. 4, (2004), pp. 1052-1068.

[9] P. Knoll, "Driving assistance systems", Brakes, Brake Control and Driver Assistance Systems, (2014), pp. $180-185$.

[10] A. Sayeed and S. A. Sadi, "Driver Drowsiness Detection using Face Monitoring and Pressure Measurement", Journal of Embedded System \& Applications, vol. 5, no. 3, (2018), pp. 12-18.

[11] T. Kundinger, A. Riener and N. Sofra, "A Robust Drowsiness Detection Method based on Vehicle and Driver Vital Data”, Mensch und Computer 2017-Workshopband: Spielend einfach interagieren, vol. 13, (2018), pp. 433-440.

[12] S. V. Kumar and A. S. Ponraj, "Automatic Driver and Vehicle Safety Monitoring System", Intelligent Embedded Systems, (2018), pp. 129-146.

[13] S. K. L. Lai and A. Craig, "A critical review of the psychophysiology of driver fatigue", Biological Psychology, vol. 55, (2001), pp. 173-194.

[14] T. Yousaf, G. Pagano, F. Niccolini and M. Politis, "Excessive daytime sleepiness may be associated with caudate denervation in Parkinson disease", Journal of the Neurological Sciences, vol. 387, (2018), pp. 220-227.

[15] J. Cheung, C. Ruoff, H. Moore, K. A. Hagerman, J. Perez, S. Sakamuri and J. Sampson, "Increased EEG Theta Spectral Power in Sleep in Myotonic Dystrophy Type 1", Journal of Clinical Sleep Medicine, vol. 14, no. 2, (2018), pp. 229-235.

[16] J. D. Wu and T. R. Chen, "Development of a drowsiness warning system based on the fuzzy logic images analysis”, Expert Systems with Applications, vol. 34, (2008), pp. 1556-1561.

[17] T. Danisman, I. M. Bilasco, C. Djeraba and N. Ihaddadene, "Drowsy Driver Detection System Using Eye Blink Patterns", IEEE International Conference on Machine and Intelligence (ICMWI), (2010), pp. 230-233.

[18] J. I. Jo, J. H. Kim and K. R. Park, "A method to classify eye status(open/close) in drowsy driver detection system", The Institute of Electronics Engineers of Korea, vol. 34, no. 1, (2011), pp. 1154-1156.

[19] J. M. Choi, H. Song, S. H. Park and C. D. Lee, "Implementation of Driver Fatigue Monitoring System", The Journal of Korea Information and Communications Society, vol. 37C, no. 9, (2012), pp. 711-720.

[20] S. M. Kang, Y. M. Yang and K. M. Huh, "Development of Drowsiness Checking System for Driver using eyes Image Histogram", Journal of Institute of control, Robotics and System, vol. 21, no. 4, (2015), pp. 300-335.

[21] B. N. Manu, "Facial features monitoring for real time drowsiness detection", Innovations in Information Technology (IIT), 2016 12th International Conference, (2016), pp. 1-4.

[22] B. G. Lee, J. H. Park, C. C. Pu and W. Y. Chung, "Smartwatch-based driver vigilance indicator with kernel-fuzzy-C-means-wavelet method”, IEEE Sensors Journal, vol. 16, no. 1, (2016), pp. 242-253. 
[23] R. K. Singh, A. Sarkar and C. S. Anoop, "A health monitoring system using multiple non-contact ECG sensors for automotive drivers", Instrumentation and Measurement Technology Conference Proceedings, (2016), pp. 1-6.

[24] Y. J. Choi, J. Y. Lee and S. H. Kong, "Driver ECG Measuring System with a Conductive Fabric-Based Dry Electrode", IEEE Access, vol. 6, (2018), pp. 415-427.

[25] M. H. Chase, "Brain electrical activity and sensory processing during waking and sleep status", Principles and Practice of Sleep Medicine, (2000), pp. 101-119.

[26] G. Li and W.-Y. Chung, "Estimation of Eye Closure Degree Using EEG Sensors and Its Application in Driver Drowsiness Detection”, Sensors, vol. 14, no. 9, (2014), pp. 17491-17515.

[27] N. B. Patil, R. P. Mirajkar, S. Patil and P. Patil, "A Method for Detection and Reduction of Stress using EEG”, International Research Journal of Engineering and Technology, vol. 4, no. 1, (2017), pp. 15981604.

[28] J. Minguillon, M. A. Lopez-Gordo and F. Pelayo, "Trends in EEG-BCI for daily-life: Requirements for artifact removal", Biomedical Signal Processing and Control, (2017), pp. 407-418.

[29] J. Minguillon, M. A. Lopez-Gordo and F. Pelayo, "Trends in EEG-BCI for daily-life: Requirements for artifact removal", Biomedical Signal Processing and Control, vol. 31, (2017), pp. 407-418.

\section{Authors}

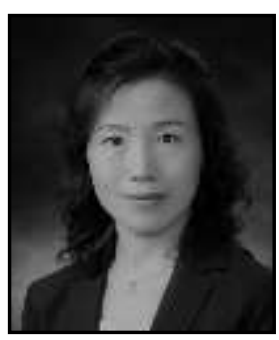

Jeonghee Hwang, she received Ph.D degrees from Chungbuk National University in 2005 in computer science. In 2006 joined the faculty of Namseoul University, where she is now an assistant professor. Her research interests include data mining, semantic web, ubiquitous computing and big data processing.

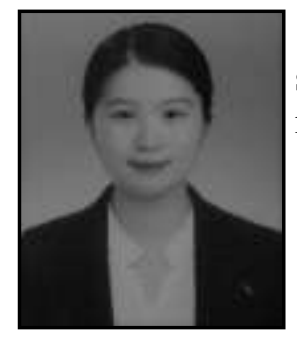

Soonwon Ahn, she received the B.S degree from department of software, Konkuk University, Seoul, Korea, in 2017. She is interested in IoT and machine learning.

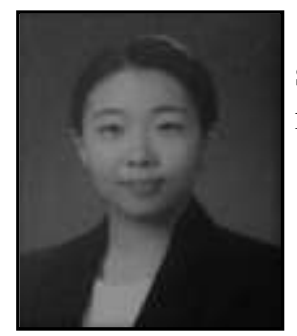

Jiyeon Kim, she received the B.S degree from department of software, Konkuk University, Seoul, Korea, in 2017. She is interested in IoT and pattern recognition.

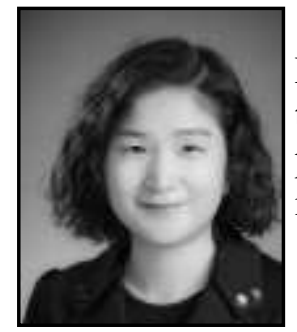

Jeonghee Chi, she received Ph.D degrees from Chungbuk National University in 2006 in computer science. In 2007 she joined the faculty of Konkuk University, where she is now an assistant professor. Her research interests include IoT, stream data mining, machine learning and big data analysis. 